\title{
Los trabajos de etnología-antropología en la revista Kalathos del Seminario de Arqueología y Etnología Turolense
}

\author{
FRANCISCO BURILLO MOZOTA \\ Seminario de Arqueología y Etnología Turolense. \\ Facultad de Humanidades y CC. SS. de Teruel
}

\section{RESUMEN}

La revista Kalathos surgió en 1981. Es el órgano del Seminario de Arqueología y Etnología Turolense (SAET), vinculado al Colegio Universitario de Teruel, hoy Facultad de Humanidades y Ciencias Sociales en la ciudad. La revista tiene una edición anual y aparece dividida en dos secciones, Arquelogía y Etnología o Antropología. Desde 1981, esta segunda sección ha tenido menor peso editorial que la primera y su ámbito de estudio ha sido casi exclusivamente la provincia de Teruel.

Palabras clave: Seminario de Arqueología y Etnología Turolense, Instituto de Estudios Turolenses, Arqueología, Etnología, Teruel.

\section{SUMMARY}

The journal Kalathos appeared in 1981. It is published annually by the Seminario de Arqueología y Etnología Turolense (SAET), an institute of Teruel College, today called School of Arts and Social Sciences, in Teruel. The journal contains two sections, Archaeology and Ethnology or Anthropology. Since 1981, this second section has been smaller than the first and been targeted, almost exclusively, at the province of Teruel.

Key Words: Seminario de Arqueología y Etnología Turolense, Instituto de Estudios Turolenses, Archaeology, Ethnology, Teruel.

En 1979 se legaliza en Teruel la Asociación Cultural: Seminario de Arqueología y Etnología Turolense (SAET). Desde su fundación se vincula al Colegio Universitario de Teruel, actual Facultad de Humanidades y Ciencias Sociales, donde tiene su sede, regularizada a partir de un convenio establecido con la Universidad de Zaragoza. Asímismo, en su desarrollo ha sido definitivo la adscripción del SAET al Instituto de Estudios Turolenses (IET), lo que ha permitido contar con un apoyo económico

RDTP, LVII, 1 (2002): 121-128 
estable, con el que consolidar las diversas actividades que se han llevado a cabo, entre ellas, cubrir parte de los costes de la revista Kalathos.

La revista Kalathos surge en el año 1981, pretendiendo una periodicidad anual. Hasta el año 2000 han aparecido 19 números, en volúmenes sencillos y dobles. Su edición ha sido posible gracias al apoyo inicial de la Diputación Provincial de Teruel y del IET. A partir de 1990 se contó con la ayuda de la Universidad de Zaragoza y para el último número se ha sumado la Caja Rural de Teruel.

El objetivo del SAET era, y es, el desarrollo de las investigaciones en materia de arqueología y etnología, tal como su nombre recoge. Por ello Kalathos aparece desde el primer número con dos secciones diferenciadas: Arqueología y Etnología, esta última sección se llamará a partir del número 5 de Antropología. De esta temática se han publicado un total de 37 artículos, aparecidos a lo largo de todos los números de la revista, a excepción del $4-5$, dedicado monográficamente a la época ibérica, y del 15 , en el que la sección fue sustituida coyunturalmente por la de Hispanocéltica.

\section{COLABORADRES}

La revista Kalathos, convertida en el órgano principal de expresión del SAET canaliza el resultado de las investigaciones realizadas por el grupo que forma esta institución cultural, con una orientación territorial destacada: la de la propia Asociación, la provincia de Teruel. Sin embargo, esta especialización no limita la publicación de investigaciones realizadas en otros ámbitos territoriales, ni tampoco la acogida de artículos elaborados por investigadores vinculados a otras instituciones. No obstante, si se comparan los trabajos publicados en Arqueología y los que aparecen en Etnología/Antropología, se desprende que el conjunto en este último apartado se halla más vinculado al grupo del SAET y al territorio turolense, que el de Arqueología. Presenta, asímismo, un número menor y con una aparición más irregular, fruto de la mayor consolidación de las actividades arqueológicas en el desarrollo del SAET. De hecho, si se analiza el número de artículos publicados sobre Antropología/Etnología, puede observarse que la mayor parte, 26, aparecen en los volúmenes anteriores al 9 , con una presencia de entre 5 y 8 trabajos por número, mientras que en la etapa más reciente sólo se publican 2 artículos por número, con presencia de uno sólo en el número 1. Esta última fase ha quedado reducida a dos de los autores más prolíficos: José Palomar Ros y Angel Gonzalvo Vallespí. 


\title{
KALATHOS 18-19
}

\author{
REVISTA
}

DEL

Seminario de Arqueología y Etnología Turolense

FTAD. DE HUMANIDADES Y CIENCIAS SOCIALES DE TERUEL

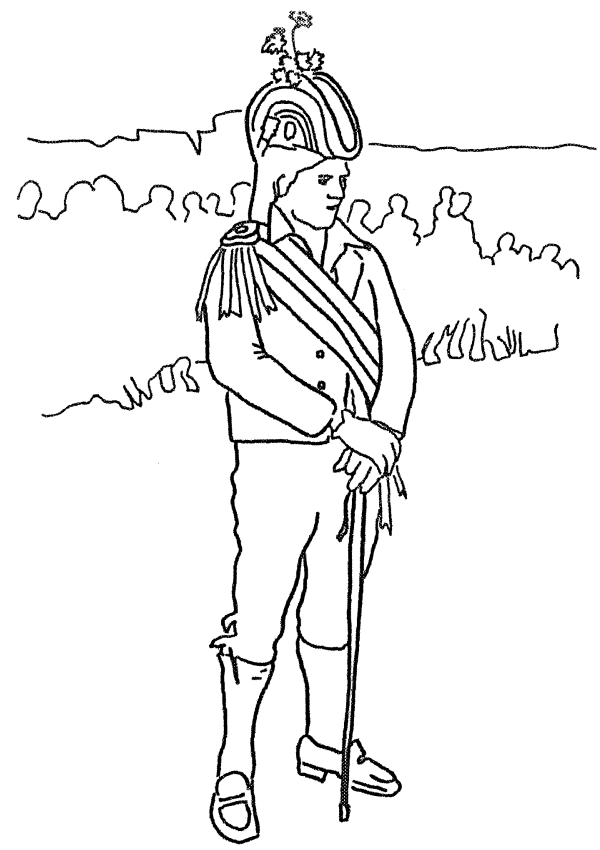

TERUEL, 1999-2000 
Hay 25 artículos en esta publicación que se adscriben a miembros, antiguos o actuales, del SAET (pongo entre paréntesis aparece el número de artículos): José F. Casabona Sebastián con E. Gargallo Monforte (1), M. ${ }^{a}$ Pilar Chinarro (1), Ángel Gonzalvo Vallespí (6), Rafael Lorenzo Alquezar (2), Monserrat Martínez González (1), José Palomar Ros (8), Jacinto M. Porro Gutiérrez (2), M. ${ }^{a}$ Elisa Sánchez Sanz (3) y junto con Antonio Gimeno Gracia (1).

Los 12 restantes son de investigadores pertenecientes a otras instituciones, con una presencia ocasional en la revista Kalathos, tal como puede deducirse del número de artículos publicados: Carmelo Lisón Tolosana (1), Garry Marvin (1), Rosario Otegui (1), Guillermo Redondo Veintemillas (1), Eulogio Soriano Lázaro (2), Trinidad Anguita Amador (1), Francesc Llop i Bayo (1), Manuel Delgado Ruiz (1), Grupo Aragonés de Estudios Tradicionales (1), José Luis Mingote Calderón (1) y Bernardo Riego (1).

\section{TEMÁTICA}

Para una mejor visión de los temas tratados he detallado toda la bibliografía aparecida en Kalathos en su sección de Antropología/Etnología agrupada por temas. Debe señalarse que existe una serie de trabajos que en otras publicaciones aparecen calificados como arquitectura popular, pero que en nuestro caso se considera histórica y aparecen dentro de la sección de Arqueología.

Puede observarse la existencia de dos grandes bloques desiguales; aquellos artículos realizados con una orientación antropológica aparecen en menor número que los que tienen una orientación etnográfica, con mayor peso de la descripción que de la interpretación. Los estudios antropológicos vienen protagonizados por C. Lisón y otros investigadores formados en su departamento universitario, como J. M. Porro, F. Llop, R. Otegui. Distinta es la orientación dada por R. Lorenzo, desde la filosofía hermeneútica.

Sin duda ha sido el tema de la literatura oral y la música tradicional turolense, con 15 artículos, el que polariza la revista, especialmente en su segunda etapa, en la que se puede considerar como tema monográfico, vinculado, por otra parte, a dos autores: J. Palomar, especializado en el tema de romances y coplas, y G. Vallespí en el de dances. El tema festivo, con 9 trabajos, es el segundo más tratado. También se centra en la provincia de Teruel y los trabajos se han agrupado según traten aspectos de tipo general, de carácter religioso, y taurino, tema este último que mereció la atención de Manuel Delgado y del investigador inglés G. Marvin. 
Referente a los estudios sobre temas artesanales, dos tratan aspectos artísticos -como los estampados de telas o la elaboración de objetos con paja- y un tercero tiene una orientación tecnológica, centrada en la fabricación de chocolate. En el campo de las actividades agropecuarias, únicamente se ha tratado el asunto de la trashumancia.

Concluye la recopilación con un inventario de las seis filmaciones inéditas que el SAET había realizado hasta 1982.

\section{Antropología y estudios de carácter general}

Anguita Amador, T. 1985-86. "Los grupos indígenas de la Patagonia". N. ${ }^{\circ}$ 5-6: 377-395.

Lisón Tolosana, C. 1985-86. "Vagad o la identidad aragonesa en el siglo XV (Antropología social e Historia).. N. ${ }^{\circ}$ 5-6: 265-307.

Lorenzo Alquezar, R. 1981. "Hombre, símbolo y cultura tradicional". N. ${ }^{\circ} 1$ : 103-112.

- 1986-86. "Antropología e imaginación. Una introducción al pensamiento de Gilbert Durand. N. ${ }^{\circ}$ 5-6: 333-354.

Llop I Bayo, F. 1987-88. "Las campanas y las horas (Del tiempo en la sociedad tradicional)m. N. ${ }^{\circ}$ 7-8: 227-251.

Porro Gutiérrez J.M. 1985-86. “Etnografía, etnología y antropología. Un intento de esclarecer el panorama de los estudios de la cultura". N. ${ }^{\circ}$ 5-6: 309-331.

- 1985-86. “El grupo 'Masoveros'. Aproximación a la identidad cultural desde la antropología. N. ${ }^{\circ}$ 5-6: 367-376.

Riego, B. 1989-90,. "La veracidad fotográfica y sus límites imaginarios. Análisis de los mitos visuales y tecnológicos del objeto fotográfico". N. ${ }^{\circ}$ 9-10: 287-309.

\section{Literatura oral y música tradicional}

Grupo Aragonés de Estudios Tradicionales (GAET). 1987-88. "Recreación literaria de la tradición oral fantástica aragonesa». № 7-8: 311-313.

\subsection{Romances, canciones y coplas}

Palomar Ros, J. 1982. "Dos versiones del romance: Doña Inés y el segador, recogidas en Valbona y Orrios (Teruel). N. ${ }^{\circ}$ 2: 177-184.

- 1987-88. “El tópico aragonés y las canciones de jota”. N. ${ }^{\circ}$ 7-8: 253-272. 
- 1989-90. "Censura y transgresión de la norma social en las coplas de Jotan. N. ${ }^{\circ}$ 9-10: 261-286.

- 1991-92. "Cantos de taberna tradicionales". N. ${ }^{\circ}$ 11-12: 399-417.

- 1993-95. "Versiones a lo divino y parodias profanas en la literatura tradicional turolense. N. ${ }^{\circ}$ 13-14: 249-281.

- 1998. "Un ejemplo de tradición oral y su transición: Aniceto Peiró (Albentosa)». N. ${ }^{\circ}$ 17: 165-215.

- 1999-00. "Misoginia o antifeminismo en las coplas (de jota)". N. ${ }^{\circ}$ 18-19: 305-341.

Soriano Lázaro, E. 1981. “El romance de la mala suegra recogido en Mezquita de Loscos". N. ${ }^{\circ}$ 1: 179- 182.

- 1982. "Romance de tradición oral recogido en Mezquita de Loscos (Teruel): Las señas del marido". N. ${ }^{\circ} 2: 151-155$.

\subsection{Dances}

Gonzalvo Vallespí, A. 1987-88. "Introducción al dance en la provincia de Teruel. N. ${ }^{\circ}$ 7-8: 281-310.

- 1991-92. "El dance de Alloza». N. ${ }^{\circ}$ 11-12: 365-398.

- 1997. "Las danzas de La Iglesuela del Cid". N. ${ }^{\circ}$ 16: 153-186.

- 1998. "El dance de Urrea de Gaén". N. ${ }^{\circ}$ 17: 133-164.

- 1999-00. "El dance de Visiedo". N. ${ }^{\circ}$ 18-19: 275-304.

\section{Fiestas populares}

Chinarro, M. ${ }^{a}$ P. 1981. "Las fiestas de Gea de Albarracín. Introducción a su estudion. N. ${ }^{\circ}$ 1: 139- 156.

Palomar Ros, M. P. 1993-95. “Los Bureos en Mora de Rubielos". N. ${ }^{\circ}$ 13-14: 201-247.

Redondo Veintemillas, G. 1981. "Fiestas populares en el siglo XVIII aragonés. Notas sobre la proclamación de Fernando III en Alcañiz (1746)". N. ${ }^{\circ}$ 1: 179- 182.

Sánchez Sanz, M. ${ }^{a}$ E. 1981. "El ciclo festivo en la provincia de Teruel. N. ${ }^{\circ}$ 1: $113-132$.

\subsection{Religiosas}

Casabona Sebastián, J. F. y E. Gargallo Monforte. 1987-88. “Sobre la romería y el santuario de La Estrella (Mosqueruela).. N. ${ }^{\circ}$ 7-8: 315-337. 
Sánchez Sanz, M. ${ }^{a}$ E. 1981. "La Semana Santa en el Bajo Aragón". N. ${ }^{\circ}$ 1: 133-138.

- 1982. "La devoción y las romerías: dos aspectos fundamentales de la religiosidad popular turolense". N. ${ }^{\circ}$ 2: 185-206.

\subsection{Taurinas}

Delgado Ruiz, M.. 1987-88. "La fiesta del toro en Terriente. Un ejemplo de campo unificado de focalización". N. ${ }^{\circ}$ 7-8: 273-280.

Marvin, G. 1982. “Una orientación para un interpretación antropológica de la fiesta del toro emboladon. N. ${ }^{\circ}$ 2: 157-175.

\section{Artesanías}

Gimeno Gracia, A. y Mª E. Sánchez Sanz. 1987-88. "Ayer y hoy del chocolate: las fábricas de Torre los Negros, Barrachina, Luco de Jiloca, Calamocha y Teruel". N. ${ }^{\circ}$ 7-8: 355-381.

Martínez González, M. 1981. "Una artesanía de estampado directo en Mosqueruela (Teruel)". N. ${ }^{\circ}$ 1: 157-178.

Mingote Calderón, J. L. 1987-88. "La utilización de la paja de cereales en el mundo rural tradicional". N. ${ }^{\circ} 7-8: 339-353$.

\section{Pastoreo}

Otegui, R. 1985-86. "Ir a extremar: Algunas prácticas de trashumancia y pastoreo en la comarca del Maestrazgo turolense". N. ${ }^{\circ}$ 5-6: 355-365.

\section{Documentos filmicos}

Gonzalvo Vallespí, A. 1982. "Filmaciones etnológicas realizadas por el Seminario de Arqueología y Etnología Turolense». N. ${ }^{\circ}$ 2: 207-208.

\section{OTRAS PUBLICACIONES}

El SAET ha desarrollado una serie monográfica que lleva el sobrenombre de Serie Etnología. Hasta el presente se han publicado ocho núme- 
ros, el primero de ellos fue una reducida edición ciclostilada. Como es obvio, las temáticas tratadas no difieren de las aparecidas en la revista Kalathos, aunque sí se amplía la nómina de autores que participan: Antonio M. Carreras Alvar, Francisco Burillo y Pilar Escuder. Las publicaciones, ordenadas por orden cronológico, son:

VV. AA. 1980. Las plantas silvestres y el hombre. Serie Etnología O. Teruel, 23 pp. y 9 cuadernillos.

Martínez, M. Y A. M. Carreras. 1981. San Antonada de Mirambel. Serie Etnología 1. Teruel, carpeta de 12 pp., y 13 láms.

Burillo, F. Y A. Gonzalvo. 1982. La fabricación de la esquila en Mora de Rubielos. Serie Etnología 2. Teruel, 60 pp., 6 láms., 14 figs.

Gargallo, E. 1982. La romería de la Estrella, Mosqueruela. Serie Etnología 3. Teruel, 62 pp., 14 figs.

Burillo, F. 1983. La alfareria de Huesa del Común. Serie Etnología 4. Teruel, 64 pp., 9 láms., 21 figs.

Palomar, J.; M. ${ }^{a}$ P. Chinarro y P. Escuder. 1985. Antología de jotas de la provincia de Teruel, I. Serie Etnología 5. Teruel, 264 pp.

Gonzalvo, A.. 1996. La Memoria Cinematográfica del Espectador. Panorámica sobre los cines en Teruel. Serie Etnología 6. Teruel, 144 pp., 90 figs.

Palomar, J. 1999. Canciones Populares de la Guerra Civil. Recogidas en la Provincia de Teruel. Serie Etnología 7. Teruel, 144 pp., 32 figs.

PERSPECTIVAS ACTUALES Y DE FUTURO

La tendencia en la publicación de la revista marca la situación actual y su desarrollo a corto plazo. El grupo de investigadores que actualmente forman el SAET orientan su atención de forma preferencial hacia la arqueología, quedando los temas de antropología/etnología limitados a la literatura oral, en concreto a los romances, coplas y dances.

No obstante, existe una tarea pendiente cuya realización se ha asumido y es la de dar a conocer las filmaciones inéditas, realizadas en la década de los ochenta, inicialmente en super-ocho y posteriormente en vídeo VHS. A pesar de las deficiencias técnicas de dichas filmaciones, somos conscientes de que se han convertido en un documento histórico, a veces único. Su edición está prevista que se realice en soporte informático. 